\title{
HYBRID SIMULATION AND OPTIMIZATION-BASED CAPACITY PLANNER FOR INTEGRATED PHOTOVOLTAIC GENERATION WITH STORAGE UNITS
}

\author{
Esfandyar M. Mazhari \\ Jiayun Zhao \\ Nurcin Celik \\ Seungho Lee \\ Young-Jun Son \\ Larry Head \\ Systems and Industrial Engineering \\ The University of Arizona \\ Tucson, AZ, 85721, USA
}

\begin{abstract}
Unlike fossil-fueled generation, solar energy resources are geographically distributed and highly intermittent, which makes their direct control difficult and requires storage units. The goal of this research is to develop a flexible capacity planning tool, which will allow us to obtain a most economical mixture of capacities from solar generation as well as storage while meeting reliability requirements against fluctuating demand and weather conditions. The tool is based on hybrid (system dynamics and agent-based models) simulation and meta-heuristic optimization. In particular, the proposed tool has been developed for scenarios, where photovoltaic generators and storage units (compressed-air-energy-storage and super-capacitors) are used to supply energy demands in a region characterized by different house-holds considering different times and seasons. The constructed tool has been used to test impact of several factors (e.g. demand growth, efficiencies in PV panel and storage techniques) on the total cost of the system. Initial results look quite promising.
\end{abstract}

\section{INTRODUCTION}

An increase in the concentrations of greenhouse gases is believed to be a major cause for climate changes in temperature (global warming), storm severity, and sea level. And, greenhouse gas emissions come primarily from the combustion of fossil fuels. For example, energy-related carbon dioxide $\left(\mathrm{CO}_{2}\right)$ emissions, resulting from the combustion of petroleum, coal, and natural gas, represented 82 percent of the total U.S. greenhouse gas emissions in 2006 <http://www.eia.doe.gov/bookshelf/brochures/greenhouse/Chapterl.htm>. To avoid damaging climate change, there is worldwide agreement on the need to reduce greenhouse emissions. For example, European Union (EU) has agreed to reduce greenhouse gases by $30 \%$ by 2020 (Freris and Infield 2008). Similarly, in March 2009, the US House of Delegates voted for the measure, which would require the state to cut greenhouse gases by 25 percent by 2020, and the Senate approved it.

To reduce greenhouse gas emissions from the combustion of fossil fuels, providing an energy share from renewable sources such as sunshine, wind, water, and bio-fuel is critical. Furthermore, energy potential from these renewable resources is enormous. For example, on average, the rate of solar radiation intercepted by the earth's surface amounts to a staggering average power of 20MW per person considering the present world population (Freris and Infield 2008).

However, unlike fossil-fueled generation, renewable energy resources are geographically distributed and highly dependent (intermittent) on the location, changing weather and climate conditions, which makes their direct control extremely challenging and requires storage units as an additional concern. Energy storage transforms an intermittent, renewable energy resource into one that has firm capacity value, and provides us with several benefits. First, excess energy stored when renewable energy resources (e.g. sun's rays) are strong can be used when the generated energy is insufficient (i.e., the sun is not shining or obscured by clouds) or sold to the grid in accordance with market prices for energy. Second, with energy storage, renewable dispatch can be controlled to reduce or avoid congestion, relieving pressure on transmission systems and reducing the need for traditional wires infrastructure. Third, many storage technologies can provide other services, such as 


\section{Mazhari, Zhao, Celik, Lee, Son, and Head}

reactive power, that can enhance grid stability, particularly in locations remote from load where large-scale renewable generation is often located (Ridge Energy Storage and Grid Services L.P. 2005).

The goal of this research is to design and develop a flexible capacity planning tool, which will allow us to obtain an optimal (most economical) mixture of capacities from renewable (in particular solar) generation units as well as storage capacities while meeting reliability requirements against fluctuating demand profiles and weather conditions. The capacity planning tool is based on hybrid (system dynamics model and agent-based model) simulation and meta-heuristic based optimization algorithm. In particular, the proposed tool is developed for two scenarios (involving different geographical scales), where photovoltaic (PV)-based solar generators and storage units (compressed-air-energy-storage (CAES) technique as well as super-capacitors) are used in an integrated manner to supply energy demands. In one of the scenario (involving smaller region), energy demands are characterized by different types of house-holds (e.g. singles, working couples, large families with many kids) considering weekdays, weekends, different times of the day, and different seasons (Xua et al. 2008).

As large-scale solar energy generation, especially in conjunction with storage units, is rare, obtaining realistic data has been quite a challenge in addition to the proposed modeling approaches. In this work, required data has been gathered from various credible sources, such as NASA Atmospheric Science Data Center $<$ http://eosweb. larc.nasa.gov $>$ and TEP <http://www.tep.com> for solar irradiance profiles, Energy Information Administration (EIA) $<$ http://www.eia.doe.gov> for energy consumption, National Renewable Energy Laboratory (NREL) $<$ http://www.nrel.gov> for energy production, <http://www.greenstar.org/ASE20Profile.htm>, FIRST <http://www.firstsolar.com>, and PB SOLAREX $<$ http: //www. solarecxo.com $>$ for cost and operational characteristics of PV panels, Ridge Energy Storage and Grid Services L.P. for cost and operational characteristics of CAES $<$ http://www.ridgeenergystorage.com>. The constructed capacity planning tool will be used to test impact of several factors (e.g. future demand growth, efficiencies in PV panel and CASE) on the total cost of the integrated generation and storage system and an optimal mixture of PV generation, fuel-based generation, and storage capacity.

\section{SCENARIOS AND UNDERLYING COMPONENTS}

\subsection{Two Scenarios with Different Scales}

In this research, two scenarios with different geographical scales are considered to illustrate the proposed simulation and optimization based tool, where the first scenario involves electricity consumption of the entire US and the second one is for a region in Tucson of Arizona. Considering these two scenarios will allow us to study different storage requirements (size, capacity, efficiency) and impacts against daily solar fluctuations (short-term) as well as seasonal fluctuations (long-term), and to concern different levels of detail in modeling. Each of these scenarios is discussed in the next sections.

\subsubsection{Scenario 1: Entire US (Abstract Model)}

In this scenario, it is assumed that only the PV-generation system (without involving fossil-based energy generation) is required to supply electricity consumption of the entire US without allowing a shortage. While this scenario may not be realistic at least for next couple of decades, it allows us to explore inter-relationships (in terms of cost and reliability) among fluctuating demands, generation capacities, and storage types and capacities. The goal of our study is to find an optimum (minimum cost) capacities of the PV-generation system and storage system given projected demand profiles, where the decision variables include the number of PV panels, storage capacity, and the initial energy level to be stored. In the simulation model (see Section 3.1) for this scenario, weekly power generation and storage are concerned based on the weekly US electricity demand and solar irradiance data. As sunshine is the only source of electricity, the system should be designed so that the sum of the generated energy and stored electricity should always exceed the electricity demand. While the designed system is not necessarily to be located at a single facility, we focus on the total amounts of generation and storage as transmission networks are not explicitly considered in our modeling. Our future research will concern enhancing the proposed models with explicit consideration of various constraints (e.g. transmission congestions, transformation (e.g. inverter) capacity), more detailed dynamics (e.g. phase synchronization), and interaction with the grid.

\subsubsection{Scenario 2: A Region in Tucson of Arizona (Detailed Model)}

This scenario concerns supplying electricity for about 1100 households without allowing a shortage in a region in Tucson of Arizona, where the sunshine is the only energy generation source. While the goal of our study is exactly same with that of scenario 2 (obtaining the optimal capacities of the PV-generation system and storage system), a major difference is that the 
electricity consumption is based on the individual households at every hour as opposed to the weekly, aggregated demand in scenario 1. In the simulation model for this scenario (see Section 3.2), the simulation time unit is set to hours (as opposed to weeks in scenario 1). In addition, each individual household is represented by an agent that has its own electricity consumption behavior over time for each particular season. Thus, the total electricity demand of the considered region is the collection of electricity consumption of all the individual households. Three different types of households are considered in this work (see Section 3.2 for more details).

\subsection{Solar Irradiance Profile}

Solar radiation (also referred to as insolation or irradiance) consists of the radiation that comes directly from the sun as well as the radiation that comes indirectly. Indirect Radiation includes: 1) the reflected radiation of an area, particularly strongly reflected radiation, i.e. areas covered by snow, 2) the radiation from the blue sky, and 3) other diffuse radiation. For the exact computation of the amount of energy which falls on a particular area, the angle of incidence between the solar radiation and the area is decisive. This changes with the time of day and year. The solar radiation is also reduced by numerous factors; even with a clear blue sky, only $90 \%$ of the total solar radiation gets through. While solar radiation $(S)$ is constant $\left(1.37 \mathrm{~kW} / \mathrm{m}^{2}\right.$ or $137 \mathrm{~mW} / \mathrm{cm}^{2}$ ) in space, it varies on earth depending on the time of day and year as well as the latitude and weather. Its maximum value on earth is between 0.8 and 1.0 kW $/ \mathrm{m}^{2}$ <http://www.solarserver.de/lexikon/solarkonstante-e.html>. In the case of Tucson of Arizona, based on the collected data, its average value is about $558.2 \mathrm{~W} / \mathrm{m}^{2}<\mathrm{http}: / / \mathrm{www}$. tep.com/Green/GreenWatts .

As mentioned in Section 2.1, the resolutions of simulation for scenarios 1 and 2 are weeks and hours, respectively. Therefore, while hourly/daily fluctuations in the solar irradiance are explicitly considered in scenario 2, aggregated (sum) weekly irradiances are considered in scenario 1. The following subsections discuss two main data resources (involving different time scales and locations) that have been used in this work, and how they have been modified for our models.

\subsubsection{NASA Atmospheric Science Data Center}

NASA Atmospheric Science Data Center is a website, which processes, archives, and distributes earth science data at NASA Langley Research Center in four disciplines of radiation budget, clouds, aerosols, and tropospheric chemistry. In this site, solar insolation data is available from 1983 to 2004 in daily values. While daily data can be easily aggregated to weekly data for our simulation model for scenario 1, it is a challenge for us to disaggregate the daily data to hourly data for our simulation model for scenario 2 (see Section 2.2.3 for details). Figure 1(a) shows the average daily solar insolation for different months of a year based on the data gathered for the period of years 1983-2004. Figure 1(b) shows daily fluctuations of solar insolation across different years (2001-2004).
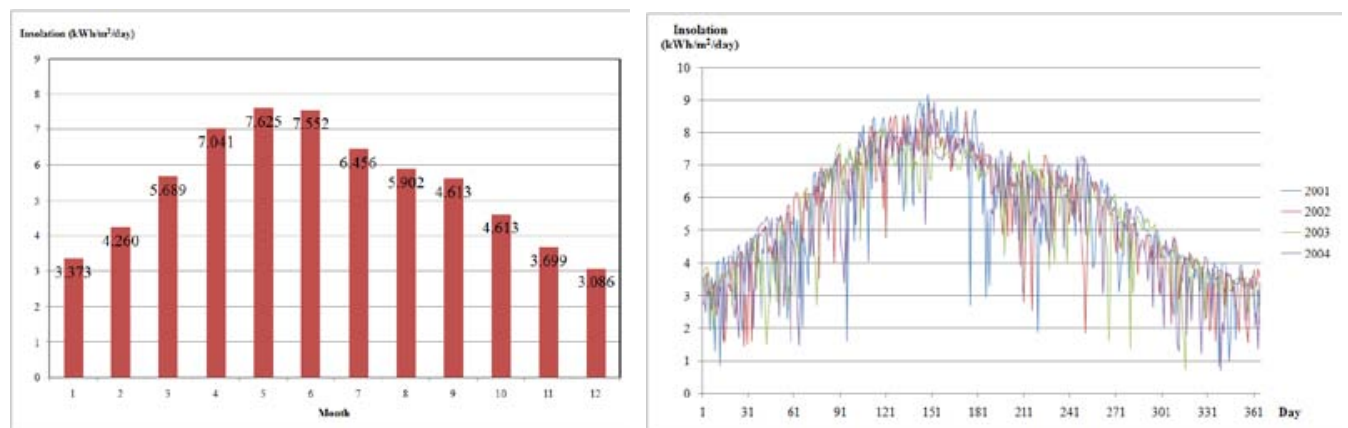

Figure 1: (a) Average daily solar insolation ( $\mathrm{kWh} / \mathrm{m}^{2} /$ day) of each month in 1983 2004; (b) Daily fluctuation of solar insolation $\left(\mathrm{kWh} / \mathrm{m}^{2} /\right.$ day $)$ across years 2001 2004

\subsubsection{Tucson Electric Power}

Tucson Electric Power (TEP) is a major utility company serving more than 375,000 customers in southern Arizona. TEP has an experimental solar energy generation site located in Springerville of Arizona, and publishes solar irradiance data of its experimental site every half an hour. Figure 2(a) shows sample data for March 21, 2009. However, their web site provides only charts (as opposed to raw data), we have manually obtained hourly data in this work, which was quite time-consuming. 

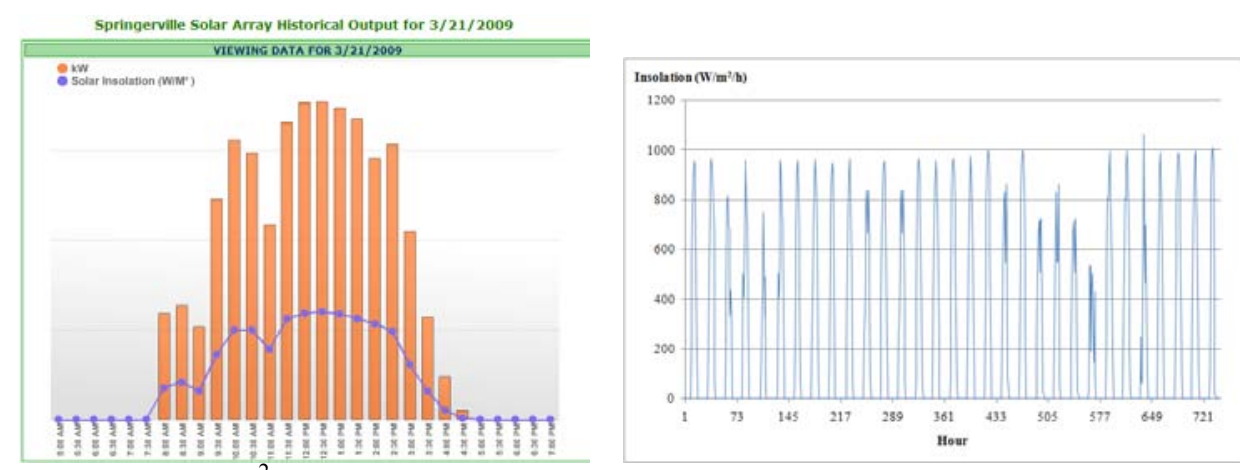

Figure 2: (a) Solar irradiation $\left(\mathrm{W} / \mathrm{m}^{2}\right)$ and generated electricity on 3/21/2009 from TEP experimental site; (b) Hourly data for solar insolation $\left(\mathrm{W} / \mathrm{m}^{2} / \mathrm{h}\right)$ for January 2009 used as a pattern

\subsubsection{Combining Data from Different Sources}

As we are interested in running a simulation with small simulation time unit (i.e. hour), we need to have hourly information for solar insolation. As mentioned earlier in Section 2.2.1.2, the hourly information is only accessible by looking up the values visually on the historical charts offered by TEP website. Thus, it is time consuming to collect a full year's hourly data manually. As an alternative solution, in this research we combined the data from NASA site (see Section 2.2.1.1) with the data from TEP (see Section 2.2.1.2). To do so, we manually extracted hourly information for two different months (January and June) and used them as base patterns to show fluctuation or variance during different days of a month. Figure 3 shows exemplary data of hourly production value and hourly solar insolation in four different days of January collected from TEP. The variation between different days is caused by environmental factors such as amount and thickness of clouds, humidity, and temperature.
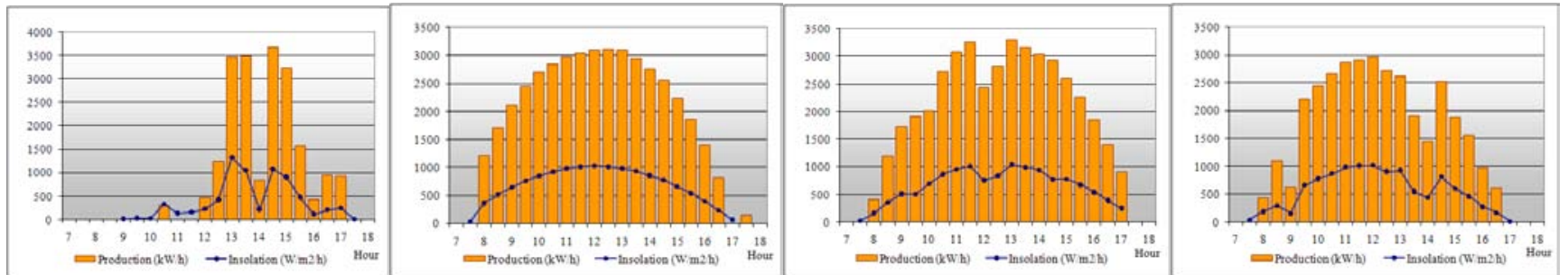

Figure 3: Hourly data for energy production $(\mathrm{kW} / \mathrm{h})$ and insolation $\left(\mathrm{W} / \mathrm{m}^{2} / \mathrm{h}\right)$ for different days

Based on the information we collected from NASA website, we calculated the average insolation per month for 22 years from 1983 to 2004 (see Figure 1). So for estimating solar insolation during individual days of months other than January and June, we assumed that the distribution of insolation during different hours in a day follows the same pattern we have for months January or June (see Figure 2(b)) with different magnitude. In other words, we assumed the insolation in each month follows the similar insolation pattern of January or June whichever closer. And we assumed that the proportion we have for monthly average value applies to the magnitude of the monthly pattern as well. For example, to find the magnitude for March, we compared the average insolation values of March with that of January. Then we applied this ratio to January pattern to build a similar hourly pattern with different magnitude. Moreover, we used a weighted average based on the time difference between the month from the January and June. Closer a month is to one of the base months, the more weight we assign to that month. For example, for March we combined the weighted average patterns of January with weight 3 with the weighted average patterns of June with weight 2. Note that 3, the weight of January, is the distance of March from June and 2, the weight of June, is the distance of March from January. In this way, we can make the closer month to have more effect on the generated pattern than a farther month. So for month March using the above mentioned method, we can build an hourly data pattern which is more similar to January pattern rather than month June, but it has the effect from both.

\subsection{Photovoltaic Generators}

There are two main technologies to convert sunlight energy to electrical energy, including 1) concentrated solar technology (CST) and 2) Photovoltaic (PV) technology. CST uses concave troughs to heat a liquid to about 700 degrees Fahrenheit to 
create steam that spins turbines used to generate power. This technology can be supplemented with natural gas backup using the same equipment. It takes between 4.5 and 5 acres of solar panel troughs for each megawatt of generating capacity.

On the other hand, in PV technology, distributed PV panels are used to produce energy. A PV panel transforms the sunlight energy into direct electrical current using the photoelectric effect. The area which includes all the PV panels is often referred to as PV-array or PV-farm. In this research, information obtained from a real PV-farm of TEP (see Section 2.2.2) is used to develop and validate our simulation models. The Springerville Generating Station Solar System (SGSSS) of TEP has a capacity of 4.6MW, which can support electricity for 727 homes and is the most productive photovoltaic array in Arizona.

Some technical details of the SGSSS are depicted in Tables 1, 2(a), and 2(b) <http://greenwatts.com/pages/SolarStats/SolarTech.html>. Information in Table 1 includes the number of PV panels available in SGSSS as well as output power and annual average production. Table 2(a) depicts three types (manufacturers) of PV panels used in the SGSSS and their technical specifications, such as output power, operating DC voltage, failure rate, among others. In Table 2(b), technical specifications of inverters (e.g. type, operating power factor, efficiency, and reliability ratios) used in SGSSS are given.

Table 1: Overview of solar generating systems in Springerville by TEP

\begin{tabular}{|c|cc|}
\hline Item & \multicolumn{2}{|c|}{ Description } \\
\hline Total Array Coverage Area: & 44 & Acres \\
\hline Number of PV Arrays - Fixed at 34 Degrees Tilt: & 34 & Arrays \\
\hline Fixed Array Azimuth Direction: & 180 & Degrees - South \\
\hline Total Number of PV Modules: & 34,980 & Modules \\
\hline Total PV DC Array Capacity Rating (STC): & 4,590 & $\mathrm{~kW}$ \\
\hline Total Inverter AC Capacity Rating: & 5,100 & $\mathrm{~kW}$ \\
\hline Total System AC PVUSA Rating: & 3,812 & $\mathrm{~kW}$ \\
\hline System Actual AC Output - 1 Minute Rating: & 5,113 & $\mathrm{~kW}(\mathrm{Dec} 6 \mathrm{~h})$ \\
\hline System Actual AC Output - 15 Minute Rating: & 4,644 & $\mathrm{~kW}$ \\
\hline Actual 2006 Annual Net AC Energy Production: & 7,765 & $\mathrm{MWh}$ \\
\hline Expected 2007 Annual Net AC Energy Production: & 7,846 & $\mathrm{MWh}$ \\
\hline Average Annual Energy Production Site Rating: & $1,730 \mathrm{kWh} / \mathrm{kW} \mathrm{DC}$ \\
\hline
\end{tabular}

Table 2: (a) PV modules used in Springerville by TEP; (b) Inverter used in Springerville by TEP

\begin{tabular}{|c|c|c|c|c|c|}
\hline Parameter: & $\mathrm{ASE}$ & First Solar & BP Solarex & Item & Description \\
\hline Number of Arrays & 26 & 4 & 4 & Inverter Type: & Xantrex PV-150 \\
\hline Module Type & $300 \mathrm{DG} / 50$ & FS-45 \& FS-50 & $\frac{\text { MST-43 }}{3000}$ & Rated Capacity: & $157 \mathrm{kVA}$ \\
\hline $\begin{array}{l}\text { Modules per Array } \\
\text { Modules per String }\end{array}$ & $\frac{450}{9}$ & $\frac{2688 \text { to } 3024}{6}$ & $\frac{3000}{5}$ & Operating Power Factor: & 0.99 \\
\hline Strings per Row & 2 & 16 to 18 & 20 & Total Harmonic Distor- & Less than $3.0 \%$ \\
\hline Power per String STC & 2,700 watts & 300 & 215 & tion: & \\
\hline Design String VOC & 595 volts & 580 volts & 545 volts & Inverter Efficiency - Aver- & $96 \%$ \\
\hline String Operating DC Volts & $380-430$ volts & $300-360$ volts & $300-310$ volts & age Daily Cycle & \\
\hline Design Array Power STC & 135,000 watts & $134,400+$ watts & 129,000 watts & Inverter Reliability in $2003 /$ & $99.92 \% / 99.87 \% /$ \\
\hline Module Failure Rate 2004 & $0.01 \%$ & Pre-Commercial & $0.09 \%$ & $2004 / 2005$ & $99.92 \%$ \\
\hline
\end{tabular}

\subsection{Energy Storage Systems}

As mentioned in Section 1, a storage system is required for renewable energy systems in order to make it dispatchable and controllable against varying load and market prices for energy. While energy storage systems can not generate energy from its original source, they are well able to store the excess energy and deliver it to the consumers when needed. They also play a critical role in adjusting the power voltages and frequencies for distribution grids. There are various types of storage systems, including 1) thermal mass systems which can store solar energy in the form of heat at useful temperatures for daily or seasonal durations, 2) molten salt systems where salt (with high specific heat capacity) is used as storage medium, 3) rechargeable batteries, 4) pumped-storage hydroelectricity systems which stores energy in the form of water pumped, 5) compressed air energy storage systems which uses pressurized air as the energy storage medium, and 6) super-capacitors. In our current research, the last two storage techniques which show quite different characteristics are considered.

\subsubsection{Compressed Air Energy Storage (CAES) Systems}

In the compressed air energy storage (CAES) systems, pressurized air is used as the energy storage medium (see Figure 4(a)). The working principle of these systems can be summarized under two main operations, compression (storage) operation and expansion (generation) operation. During the compression operation, electricity is used to compress and pump the air into an 


\section{Mazhari, Zhao, Celik, Lee, Son, and Head}

un- insulated underground storage reservoir using a group of compressors. Assuming that ideal gas law (i.e., $P V=n R T$ ) holds for gases regardless they are ideal or not, for a constant volume, as the pressure of air increases, its temperature increases as well. In CAES systems, inter-coolers and after-coolers are employed to reduce the temperature of the stored air. This way, required storage volume (i.e. cavern size) is reduced, and compression efficiency is increased. During the expansion operation, stored air is released from the storage using a group of turbines to re-generate energy. Generally, these turbines are first fired using either natural gases or distillate fuel. The best places for large scale CAES systems are identified to be mined salt caverns, hard rock and aquifers.

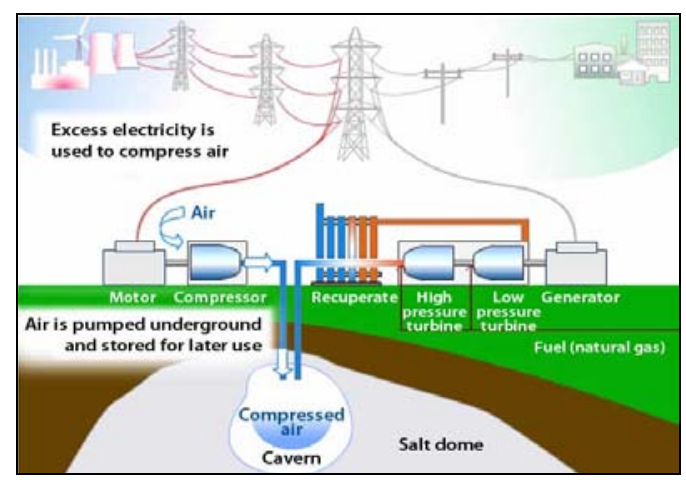

\begin{tabular}{|c|c|c|c|}
\hline Appliance & Quantity & Rated Power (Watt) & Time Period(s) \\
\hline AC & 2 & 1500 & $12 \mathrm{am}-6 \mathrm{am}, 10 \mathrm{am}-12 \mathrm{am}$ \\
\hline Fan & 5 & 70 & $8 \mathrm{am}-9 \mathrm{am}, 12 \mathrm{pm}-1 \mathrm{pm}, 5 \mathrm{pm}-9 \mathrm{pm}$ \\
\hline Refrigerator & 1 & 100 & All day \\
\hline TV & 2 & 133 & $9 \mathrm{am}-11 \mathrm{am}, 2 \mathrm{pm}-4 \mathrm{pm}, 5 \mathrm{pm}-7 \mathrm{pm}, 9 \mathrm{pm}-11 \mathrm{pm}$ \\
\hline Lights & 10 & 40 & $6 \mathrm{pm}-12 \mathrm{am}$ \\
\hline Microwave oven & 1 & 1000 & $7 \mathrm{am}-7: 30 \mathrm{am}, 11: 30 \mathrm{am}-12: 00 \mathrm{pm}, 5: 30 \mathrm{pm}-6: 30 \mathrm{pm}$ \\
\hline Coffee maker & 1 & 1000 & $7 \mathrm{am}-7: 15 \mathrm{am}, 1: 30 \mathrm{pm}-1: 45 \mathrm{pm}$ \\
\hline Dishwasher & 1 & 1800 & $1 \mathrm{pm}-2 \mathrm{pm}, 7 \mathrm{pm}-9 \mathrm{pm}$ \\
\hline Personal computer & 1 & 120 & $6 \mathrm{pm}-11 \mathrm{pm}, 3 \mathrm{pm}-4 \mathrm{pm}$ \\
\hline Monitor & 1 & 150 & $8 \mathrm{pm}-11 \mathrm{pm}, 3 \mathrm{pm}-4 \mathrm{pm}$ \\
\hline Laptop & 1 & 50 & $2 \mathrm{pm}-4 \mathrm{pm}, 9 \mathrm{pm}-11 \mathrm{pm}$ \\
\hline VCRDVD & 1 & 20 & $6 \mathrm{pm}-7 \mathrm{pm}$ \\
\hline Toaster oven & 1 & 1225 & $6 \mathrm{am}-6: 20 \mathrm{am}, 7 \mathrm{am}-7: 15 \mathrm{am}$ \\
\hline Toaster & 1 & 1400 & $9: 30 \mathrm{am}-10: 30 \mathrm{am}$ \\
\hline Clothes Dryer & 1 & 5000 & $9 \mathrm{am}-9: 30 \mathrm{am}$ \\
\hline Clothes Washer & 1 & 500 & $7 \mathrm{pm}-8 \mathrm{pm}$ \\
\hline Water heater & 1 & 5500 & . \\
\hline
\end{tabular}

Figure 4: (a) Compressed-air energy storage (Ridge Energy Storage and Grid Services L.P, 2005); (b) Sample electricity consumption for a family with 5 children

In our study, CAES system requirements are based on three main parameters: the storage capacity based on the storage volume requirement, initial storage level, and corresponding cost which are defined in detail in Section 3.4. Succar and Williams (2008) proposed a formula representing how much electrical energy can be generated per unit volume of storage cavern capacity $\left(\mathrm{E}_{\mathrm{GEN}} / \mathrm{V}_{\mathrm{S}}\right)$ (see Equation 1$)$.

$$
\frac{E_{G E N}}{V S}=\frac{\alpha}{V S} \int_{0}^{t} \dot{m}_{A}\left(\beta+1-\left(\frac{p_{b}}{p_{2}}\right)^{\frac{k_{2}-1}{k_{2}}}\right) d t
$$

In Equation 1, $E_{G E N}$ is the electrical output of the turbine and is defined by $E_{G E N}=n_{m} \cdot n_{G} \cdot \int_{0}^{t} \dot{m}_{T} w_{C V, T O T} d t$. The integral here is the mechanical work generated by the expansion of air and fuel in the turbine. $n_{m}$ is the mechanical efficiency of the turbine which reflects turbine bearing losses, $n_{G}$ is electric generator efficiency, $w_{C V, T O T}$ is total mechanical work per unit mass generated in this process and defined by $w_{C V, T O T}=w_{C V 1}+w_{C V 2}=-\int_{p 1}^{p 2} v d p+\int_{p 2}^{p b} v d p$. Further, $\dot{m}_{T}=\dot{m}_{A}+\dot{m}_{F}$ is air mass flow rate and $t$ is the time required to deplete a full storage reservoir at full output power. In Equation 1, open forms of $\alpha$ and $\beta$ are $\alpha=n_{m} \cdot n_{G} \cdot c_{p 2} T_{2}\left(1+\frac{\dot{m}_{F}}{\dot{m}_{A}}\right)$ and $\beta=\frac{c_{p 1} T_{1}}{c_{p 2} T_{2}}\left[1-\left(\frac{p_{b}}{p_{2}}\right)^{\frac{k_{2}-1}{k_{2}}}\right]$, respectively.

\subsubsection{Super-capacitors}

In super-capacitors, electrical energy is stored in the electric field between two electrodes as medium of ions. Ions allow for quick storage and release of energy (i.e., in seconds) and the super-capacitors have very high cycle times allowing them to be cycled millions of times without losing any efficiency. Also, they enable high granularity distribution of energy storage systems in contrast to centralized CAES systems. Even a single household can attach a dedicated super-capacitor to its small scale solar generation system. However, super-capacitors also possess some drawbacks. First, they have a considerable low energy density compared to those of batteries; thereby they usually store about $1 / 10^{\text {th }}$ of the energy that an electrochemical battery can hold. Second, the super-capacitors cells only allow for low voltages which require serial connections between multiple cells in order to obtain higher voltages. Third, super-capacitors have a high rate of self-discharge compared to that of an electrochemical battery. On the other hand, as a means of centralized energy storage, compressed air energy storage 
(CAES) systems are known as the most cost efficient energy storage systems. Its effect grows significantly when considered system becomes larger in scale. It also facilitates the control over the distribution of energy in conjunction with the energy generation system.

\subsection{Aggregated Demand based on Different Classes of Households}

As demand drives the electricity generation as well as storage, it is one of the major aspects of our simulation model. As mentioned in Sections 2.1, demand profiles are modeled in two different ways. In the simulation model for scenario 1, the demand is based on the monthly energy consumption (which is uniformly distributed to weekly consumption within each month) of the entire US provided by Energy Information Administration (EIA). On the other hand, the demand in the model for scenario 2 is based on the hourly energy consumptions of every individual household in the region. To this end, as part of this work, we have developed hourly power consumption profiles for three different (representative) types of households considering major appliances used by them at different times of the day. Three household types considered in this research are 1) a single working person, 2) families of a working couple, and 3) families with five children. Figure 4(b) depicts electricity consumptions for a family with 5 children (data for the other two types are not shown explicitly due to the space limit). In our simulation model (for scenario 2), the above mentioned three types of households are replicated to create the total number of residents, and the total demand of electricity is the collection of electricity demanded by all the households.

\section{PROPOSED MODELING APPROACH}

This section explains the proposed hybrid-simulation based meta-heuristic optimization model, which enables us to obtain the optimal configuration of the energy generation and storage systems. The energy system considered in this research is inherently a large scale, dynamic system containing many sub-systems such as power generation system, storage system, power grid distribution system. In general, such a large-scale system can be simulated in an aggregated manner using systems dynamics (SD) modeling method to study its overall behavior in a long run. However, it can also be simulated in a greater detail using agents-based (AB) model to analyze individual behaviors of small players as well as a system behavior based on the collection of individual behaviors. In our research, only the SD modeling method is used to construct a simulation model for scenario 1. However, both SD (for generation and storage segments) and AB methods (for demand segment) are used in a hybrid manner to construct our simulation model for scenario 2. Employing hybrid modeling methods to develop models with different levels of details has allowed us to realize different levels of required efforts in data collection and model development, and to analyze the impacts of levels of details on the simulation results. More details about each of the simulation models and their optimization models are discussed in the following subsections.

\subsection{Model for Scenario 1 (Abstract Model)}

In this model, we have employed SD modeling technique to mimic the electricity system for the entire US (see Section 2.1.1), whose simulation execution does not involve high computational power. Figure 5 depicts the screen capture of the developed simulation model in AnyLogic software. As mentioned in Section 2.1.1, this model provides weekly power generation and storage based on the weekly demand and solar irradiance data. In our work, it has been assumed that each PV unit works 7 days a week, 8 hours a day with the power generation capacity of $200 \mathrm{~W}$. Then, the generation capacity of the $\mathrm{PV}$ unit (to reflect the variation of solar irradiance over months) is varied in each month by multiplying $0.44,0.6,0.74,0.89$, $1,0.93,0.88,0.85,0.76,0.7,0.5,0.44$ for months January to December, respectively. These values have been calculated by dividing the average daily solar insolation for a specific month based on information gathered from NASA data source for the time period 1983-2004 (see Figure 1(a)) by that of month May (involving the maximum solar insolation). In other words, each value shows the ratio of daily solar insolation in a particular month with respect to the maximum daily solar insolation for a month. For example, this ratio for May is 1.00 , and the ratios for January and December (involving the least solar insolation) are $0.44(=3.373 / 7.625)$ (see Figure 1(a)).

The monthly demand variation of the electricity is estimated based on the US annual consumption over the time period of September 2007 to August 2008 provided by Energy Information Administration (EIA) (Hainoun 2009). In our simulation model, an empirical function has been developed to depict the monthly energy consumption of the entire US over a year, where the function is based on the ratio of demand of a specific month with respect to demand of month July (involving the highest rate of demand, see Figure 6(a)). Due to the physical limitations on the transmission lines, the model considers the limitation of electricity transmission among power generation, storage, and demand by constraining the maximum flow rate. Finally, CAES system has been implemented as the storage system, where its efficiency varies between $65 \%$ and $85 \%$. As shown at the bottom right corner of Figure 5, this model has been designed to be flexible to incorporate various situations by 
adjusting most of the parameter values (e.g. demand growth, PV unit cost, PV unit efficiency, storage cost, storage efficiency) as well as decision variables (e.g. generation capacity, storage capacity, and initial storage level).

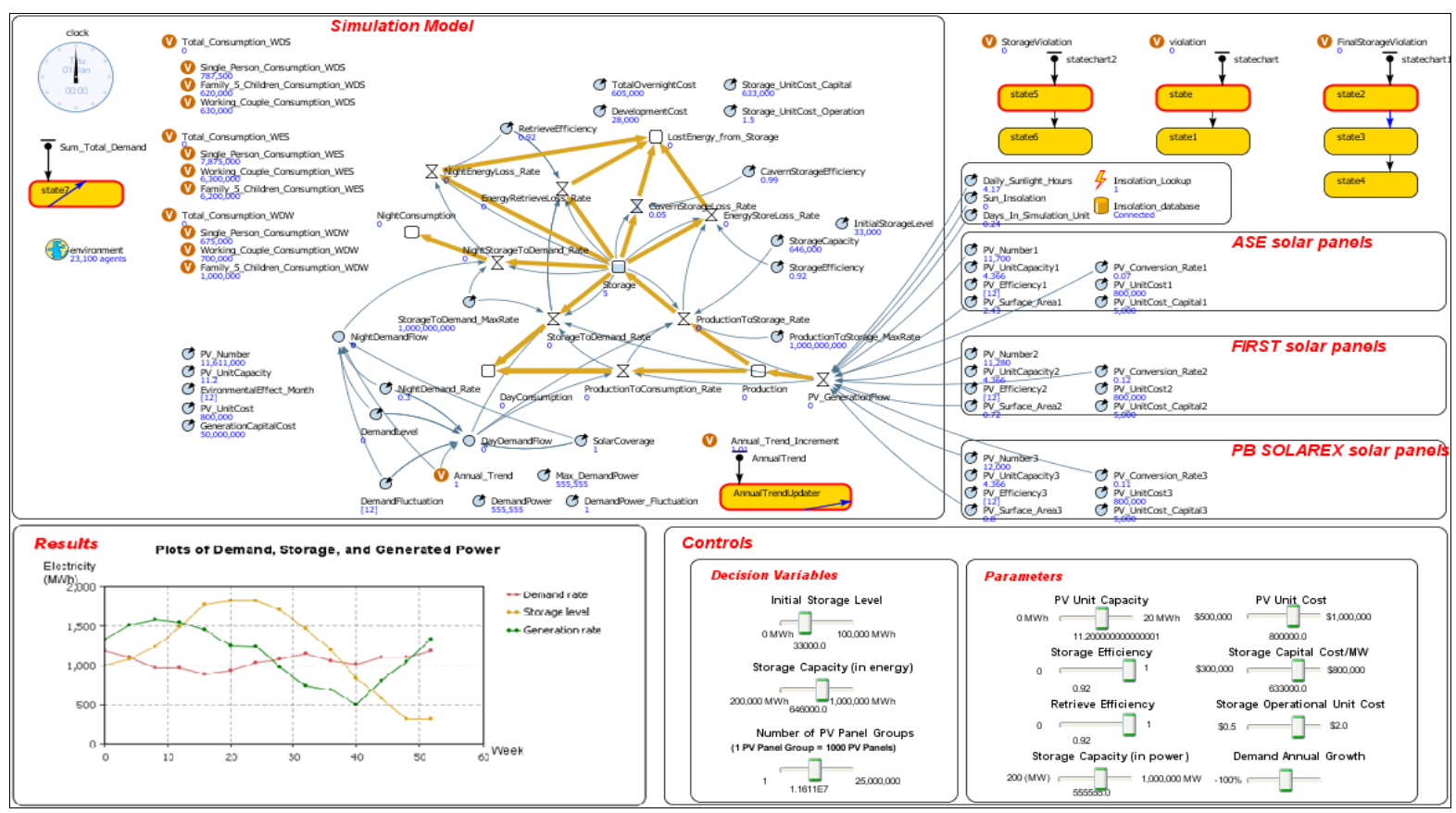

Figure 5: Snapshot of developed, flexible simulation model

\subsection{Model for Scenario 2 (Detailed, Hybrid Model)}

In this model, we have employed a hybrid modeling paradigm involving SD and $\mathrm{AB}$ techniques to simulate an electricity demand based on the electricity consumption of individual household in every hour time frame. As mentioned in Section 2.5, an agent in the $\mathrm{AB}$ model represents each of three different types of individual household and mimics its electricity consumption behavior. As mentioned earlier, three types of household are 1) a single working person, 2) family of a working couple, and 3) family of couple with five children (see Figure 4(b) for household specific demand profile). Each of them has been replicated for 500,400, and 200, respectively, to result in 1'100 households in the simulation model. During the simulation run, while the $\mathrm{AB}$ portion calculates a total demand collecting consumptions of each individual household, the SD portion simulates the interaction among the power generation, storage, and demand, which is similar to the model for scenario 1 (see Section 3.1). In order to enhance the validity of our simulation model, all the parameters in this model are based on the data obtained from the Springerville Generating Station Solar System (SGSSS) (see Section 2.3). Since the model is designed to produce hourly results, we have used the hourly solar insolation data to simulate the PV power generation (see Section 2.2.3).

\subsection{Validation of Proposed Simulation Models}

In order to validate the proposed simulation models, we have compared the generation rate and demand rate yielded from the simulation model for scenarios 1 (see Figure 6(b)) against the real US annual consumption data provided by Energy Information Administration (EIA) and electrical energy production provided by National Renewable Energy Laboratory (NREL) (see Figure 6(a)). As shown in Figure 6, the outputs obtained from the developed simulation model are appreciably close to the real data. Furthermore, the average electricity demand of agents in our detailed simulation model (for scenario 2) has been found to be about $15 \mathrm{MWh}$ per year, which confirms with the average demand of the entire households of the US (about 10 MWh per year). In our future work, we will enhancing the validity of the proposed simulation models with real consumption data of individual household (the authors of this paper are about to start a project with a utility company, which will provide them with real, individualized consumption data for different regions). 


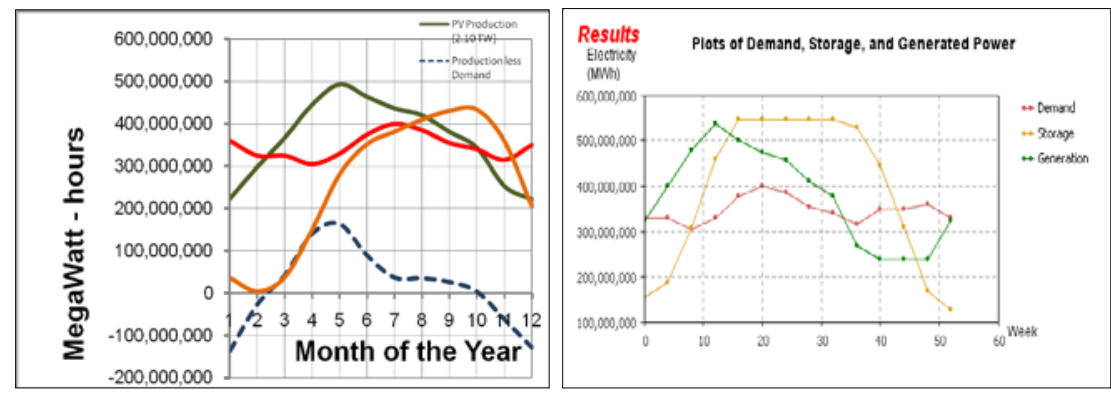

Figure 6: Comparison of (a) real data from EIA and NREL vs. (b) results generated simulation

\subsection{Meta-heuristic Optimization}

The simulation models that we have constructed (see Sections 3.1 and 3.2) are used to find a best solar system configuration involving the minimum cost without causing a blackout. To this end, we have employed OptQuest tool (Glover et al. 1999), which incorporates a combination of three meta-heuristics including Scatter Search, Tabu Search, and Neural Networks.

Equation 2 shows the mathematical formulation of the objective function in the considered optimization problem, where $N_{p v}$ is the number of PV modules, $C_{p v}$ is the unit cost of PV modules, $C_{g}^{\text {capital }}$ is the capital cost of generation, $S_{c}$ is the storage capacity, $C_{s}^{\text {capital }}$ is the unit capital cost of storage, $E_{T}$ is the energy transform rate, $E_{I S}$ is the initially stored energy, $C_{s}^{\text {operational }}$ is the unit operational cost of storage, $R^{p d}$ is the energy transition rate from production to demand, $R_{D}^{s d}$ is the daytime energy transition rate from storage to demand, $R_{D}^{d}$ is the day-time energy demand rate, $R_{N}^{s d}$ is the night energy transition rate from storage to demand, $R_{N}^{d}$ is the night energy demand rate, $S_{c}$ is storage capacity, $S_{I}$ is initial storage level and $S$ is the energy storage.

$$
\begin{aligned}
& \operatorname{Min}\left(N_{p v} \cdot C_{p v}+C_{g}^{\text {capital }}+S_{C} \cdot C_{S}^{\text {capital }}+\left(\sum E_{T}+E_{I S}\right) \cdot C_{S}^{\text {operational }}\right) \\
& \text { s.t. } \quad R^{p d}+R_{D}^{s d} \geq R_{D}^{d}, \quad R_{N}^{s d} \geq R_{N}^{d}, \quad S_{C} \geq S_{I}, \quad S \geq 0
\end{aligned}
$$

In this work, the considered total cost in the objective function (Equation 2) consists of the solar energy generation cost and the energy storage cost where their corresponding values are assumed based on real case studies and real market prices. The generation cost is further consisted of the capital cost involving land cost, hardware costs and construction costs, and the operational cost, which depends on the number of PV modules installed. Here, as the land cost and construction cost sections of capital cost is assumed not to be significantly affected by the varying number of PV modules, a fixed value for these sections of capital cost is used in this study. And the cost of PV modules is depicted as operational cost of generation and is derived from the weighted average of the market prices of two brands of PV modules those are Mitsubishi PV-UD180MF5, 180 Watt Nominal Solar Module <http: / /www. Solarharmony.com> with a selling price of $\$ 835$ and BP Solar SX3200B, 200 watt PV Module <http://www.affordable-solar.com/bp-solar-sx3200b-watt-solar-panel.htm> with a selling price of $\$ 835$. The PV module that is considered in our model has a capacity of 200 watts, and its selling price is assumed to be $\$ 800$ considering the fact that massive usage of these modules will reduce the market price.

Similarly, the cost of solar energy storage is consisted of capital (fixed) and operational (variable) costs. During when compressed air storage is considered as storage technique, the capital cost includes the equipment, land and construction cost, and the operational cost includes the maintenance and natural gas costs used for the turbine utilization. The turbines are used when transforming the electrical power into compressed air (compressing) and transforming back the stored compressed air into electricity (expansion). Therefore, the operational cost is also called as the transforming cost that depends on the total amount of energy transformed in the system. In this case, the cost of energy storage is based on the case study of Ridge Energy Storage and Grid Services L.P (2005), where the considered CAES system can enable a 50-hour supply of 270MW output power. Here, the capital cost is denoted in terms of power (in units of dollar per KW), while the operational cost is denoted in terms of energy (dollar per MWh). In our model, the storage system is comprised of a set of storage units each with a capacity of 270MW enabling 50-hour supply. The required output for the storage system is found by dividing the storage capacity (a decision variable in our objective function) by 50 hours of operation. As a result, the capital cost of storage is the unit capital cost (a total of $\$ 633 \mathrm{~K}$ per MW) multiplied by the required total output power in our model and the operational cost is assumed to be $\$ 1.5$ per MWh multiplied by the sum of total transformed and initial stored energy in the system.

When the super-capacitors are used for storage units, the main portion of the cost comes from the price of supercapacitor packages and its connection and transition. The cost of connection and transition are considered as operational 


\section{Mazhari, Zhao, Celik, Lee, Son, and Head}

costs. However in our simulation model this cost is not considered as it is quite negligible as compared to the cost of supercapacitor packages. On the other hand, the price of each super-capacitor is based on the market price information of Maxwell $B C A P 350$ (Simjee and Chou 2008) where the price of each unit of super capacitor is about $\$ 20$ with the unit maximum capacitance of 1100 Jules. Therefore, in our model, the capital cost of super capacitors is $\$ 65 \mathrm{M}$ per MWh.

The proposed optimization model (see Equation 2) has three decision variables, including the number of PV modules $\left(N_{p v}\right)$, storage capacity $\left(S_{c}\right)$, and initial storage level $\left(E_{I S}\right)$. The number of PV modules determines the operational cost of the electric power generation. The storage capacity, which indicates the maximum amount of energy can be stored in the storage system, determines the total capital cost of storage. Finally, the initial storage level is related to the storage operational cost.

The proposed optimization model (see Equation 2) contains three constraints based on the requirement in the considered scenario, where the sum of the generated energy and stored electricity should always exceed the electricity demand. The first constraint in Equation 2 regulates the relationship among solar energy generation, storage, and demand, where the demand can be fulfilled by energy generation, storage, or both. As the day-time demand and night-time demand are separated in this model, their constraints are also built separately as shown in the first and second constraints in Equation 2. The third constraint in Equation 2 guarantees that the initially stored energy is less than or equal to the total storage capacity. Usually, a certain amount of energy is needed at the beginning of the simulation run (e.g. January) to guarantee finding a feasible solution without allowing a shortage against fluctuating demand of the future. The last constraint in Equation 2 is to ensure the amount of stored energy is not allowed to be negative at any time during the simulation.

\section{EXPERIMENT AND SENSITIVITY ANALYSIS}

In this section, we discuss sensitivity analyses conducted using the proposed simulation and optimization models discussed in Section 3. In our research, we test the effect of demand, storage efficiency, and PV efficiency on the capital and operational costs of the solar power generation and storage system.

\subsection{Effect of Demand Increment Rate}

The goal of this experiment is to analyze the impact of the demand growth on the total cost of the solar generation and storage. Figure 7(a) depicts the increment rate of the cost compared with the cost for the stationary demand for cases with varying annual increment rates $(5 \%, 10 \%, 15 \%, 20 \%$ and $25 \%)$. Although $25 \%$ of annual increment in demand may not be realistic, it was also considered to see the effect of increment more clearly. In this experiment, the storage efficiency and PV efficiency are fixed as $85 \%$ and $17 \%$, respectively in all the comparisons. The results reveal that the cost of meeting the demand with annual increment rate is growing exponentially. In other words, when the annual demand increment rate is increased from $10 \%$ to $20 \%$, the increase in the total cost of meeting their corresponding demand becomes more than double.

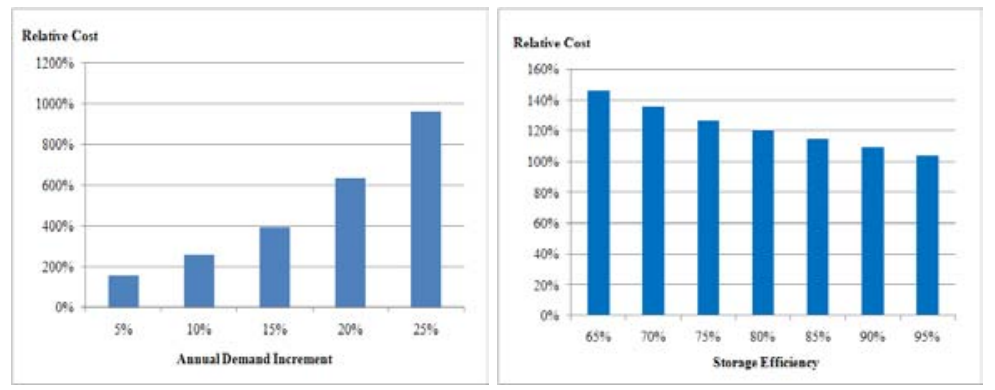

Figure 7: (a) Effect of future demand growth (annual) on the total cost vs. (b) Storage efficiency on the total storage cost

\subsection{Effect of Storage Efficiency}

The goal of this experiment is to test the effect of storage efficiency on the storage cost of the solar energy system. The storage efficiency defines the amount of energy that is lost in the energy conversion occurred during the compression (storing) and expansion (retrieving) operations in the storage system. As the storage efficiency is higher, less energy is lost during these conversions. In this experiment, the demand incremental rate and the PV efficiency are fixed as 0 and $17 \%$, respectively. Figure 7(b) reveals that the relative total cost (when the storage efficiency is $100 \%$ ) decreases as the storage efficiency increases. It is because while we can save the operating cost of the storage system with increased efficiency, the capital cost that takes the major portion of total cost remains same. 


\subsection{Effect of PV Panel Efficiencies}

The goal of this experiment is to test the impact of the PV panel efficiencies on the total cost of the solar energy system. Figure 8(a) depicts the relative cost (cost/cost when the PV efficiency is 17\%) of the systems with varying PV efficiencies. In this experiment, the demand incremental rate and the storage efficiency are assumed to be 0 and $85 \%$, respectively. Moreover, we assumed that the prices of PV panel are same for all the PV panels with different efficiencies. As the efficiency of the PV panel increases, the total system cost decreases as a less number of PV panels are needed for generating the same amount of electricity. However, the amount of reduced cost is less significant for the higher PV efficiencies. This is because in some cases the storage capacity increases (via the optimization, see Section 3.4) for the cases with increased PV efficiency.
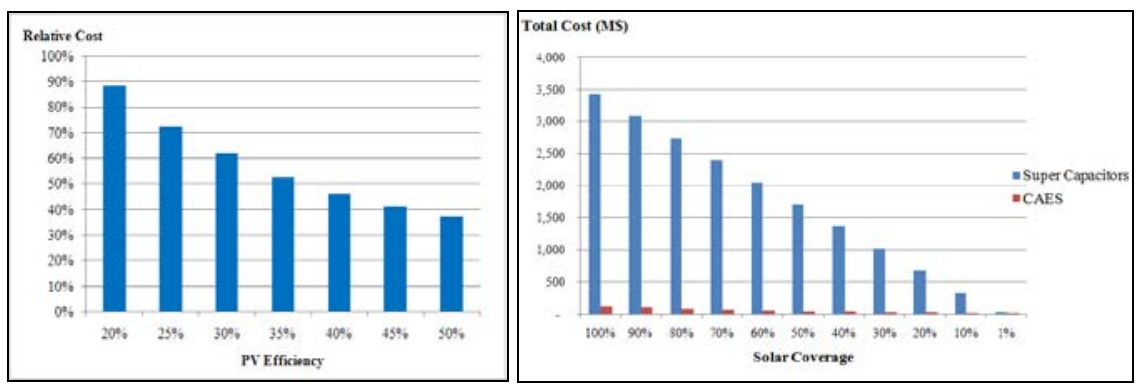

Figure 8: (a) Impact of PV efficiencies on the cost of energy system; (b) Cost comparison of CAES vs. super-capacitors

\subsection{Cost Comparison of CAES Technique vs. Super-capacitors}

The goal of this experiment is to compare CAES and super-capacitors in terms of on the total cost. Figure 8(b) depicts the total cost of the system with varying solar coverage. In this experiment, storage efficiencies for CAES and super-capacitors are assumed to be $85 \%$ and $98 \%$, respectively. As the solar coverage decreases from $100 \%$ to $1 \%$, the total cost for both technologies decreases, but in all cases, CAES is significantly more cost effective than the super-capacitors based on the current costs. This is because of the relatively large storage capacity considered in this research. However, for systems requiring highly distributed storage systems with smaller capacity, the super-capacitors are expected to be more cost effective.

\section{CONCLUSION AND FUTURE RESEARCH}

In this work, a flexible simulation has been successfully developed for integrated solar generation systems involving PV generators and storage units (CAES and super-capacitors) considering highly intermittent nature of solar energy resources. This simulation has been then used in conjunction with meta-heuristic optimization to obtain a most economical mixture of capacities of solar generation and storage while avoiding blackout against fluctuating demand and weather conditions. For two scenarios involving different geographical scales, the proposed simulation and optimization models have been successfully used to obtain an optimal mixture of required capacities of the systems. In addition, experiments were conducted using the proposed models, and the results revealed that future demand growth, storage efficiency, PV efficiency, and types of storage technologies significantly affects the total cost of the solar system. Therefore, the capacity of the solar system has to be carefully determined based on these factors. To the best of our knowledge, this is the first research work on the development of flexible simulation and optimization tools based on the real data. In our future works, the current simulation/optimization models expanded to include grid, smart-grid, other types of storage technologies, and other types of renewable energy resources such as wind and bio-fuels.

\section{ACKNOWLEDGEMENTS}

This work was supported by the Arizona Research Institute for Solar Energy (AzRISE).

\section{REFERENCES}

Energy Information Administration, Official Energy Statistics from the U.S. Government. 2008. Green House Gases, Climate Change, and Energy. Available via

<http://www.eia.doe.gov/bookshelf/brochures/greenhouse/Chapterl.htm> 
Freris, L., and D. Infield. 2008. Renewable Energy in Power Systems. John Wiley and Sons, West Susses, United Kingdom. Hainoun, A. 2009. Construction of the hourly load curves and detecting the annual peak load of future Syrian electric power demand using bottom-up approach. International Journal of Electrical Power and Energy Systems, 31(1): 1-12.

Glover, F., J. Kelly, and M. Kaguna. 1999. The OptQuest Callable Library User's Documentation. Boulder, Colorado: Optimization Technologies Inc.

Ridge Energy Storage and Grid Services L.P. 2005. The Economic Impact of CAES on Wind in TX, OK, and NM, Final Report for Texas State Energy Conservation Office. Available via <http://www.seco.cpa.state.tx.us/zzz re/re wind_projects-compressed2005.pdf>

Simjee, F. I., and P. H. Chou. 2008. Efficient Charging of Supercapacitors for Extended Lifetime of Wireless Sensor Nodes. IEEE Transactions on Power Electronics, 23(3): 1526-1536.

Succar S., and R. H. Williams. 2008. Compressed Air Energy Storage: Theory, Resources, And Applications For Wind Power, Final Report for Princeton Environmental Institute at Princeton University. Available via $<$ http://www.princeton.edu/ cmi/research/Capture/Papers/SuccarWilliams_PEI_CAES_20 08April8.pdf>

Xua, M., Z. Hub, J. Wua, and Y. Zhoua. 2008. A hybrid society model for simulating residential electricity consumption, International Journal of Electrical Power and Energy Systems, 30(10): 569-574.

\section{AUTHOR BIOGRAPHIES}

ESFANDYAR MAZHARI is a Ph.D. student in the Department of Systems and Industrial Engineering at U. of Arizona. He received his B.S. in Industrial Engineering from Tehran Poly Technique University in Iran in 1999 and M.S. in SocioEconomical Systems Engineering from Iran University of Science and Technologies in 2002 and M.S. in Systems and Industrial Engineering from U. of Arizona in 2008. He can be reached by email at <emazharieemail.arizona.edu>.

JIAYUN ZHAO is a Ph.D. student in the Department of Systems and Industrial Engineering at the U. of Arizona. She received her B.S. in Management Information System from Nanjing College for Population Program Management in Nanjing, China in 2006 and M.S. in Management Information System from U. of Arizona in 2008. She can be reached by email at <melodyun@email.arizona.edu>.

NURCIN CELIK is a Ph.D. candidate in the Department of Systems and Industrial Eng. at U. of Arizona (UA). She received her B.S. in Industrial Eng. from Bogazici University, Turkey and M.S. in Systems and Industrial Eng. at U. of Arizona. She has received several awards such as the IIE Outstanding Graduate Research Award (2009) and the Best Ph.D. scientific poster award in the Ph.D. Colloquium at IERC2009. She serves as the treasurer of INFORMS Student Chapter at UA and a student member of IIE, INFORMS and SME. She can be reached at<nurcinkoyuncuegmail.com>.

SEUNGHO LEE is a Ph.D. candidate in the Department of Systems and Industrial Engineering at U. of Arizona. He received his BEng in Industrial Engineering from Korea University in Korea in 1999 and his MS in Industrial Engineering from Texas A\&M University in 2005. His research focuses on application of distributed simulation and simulation of human decision-making. $\mathrm{He}$ is a student member of IIE and INFORMS. $\mathrm{He}$ can be reached at <mountleedemail.arizona.edu>.

YOUNG-JUN SON is an Associate Professor of Systems and Industrial Engineering at U. of Arizona. He is an associate editor of the Int. Journal of Modeling and Simulation and the Int. Journal of Simulation and Process Modeling. He has received the SME 2004 Outstanding Young Manufacturing Engineer Award, the IIE 2005 Outstanding Young Industrial Engineer Award, IERC Best Paper Awards (2005 in modeling and simulation; 2008 in homeland security), and the Best Paper of the Year Award in 2007 from Int. Journal of Industrial Engineering. $\mathrm{He}$ can be reached by email at <sonesie.arizona.edu>.

LARRY HEAD is the Department Head and an Associate Professor of Systems and Industrial Engineering at U. of Arizona. He was a Senior Vice President and Director of Research and Development at a business unit of Siemens ITS. His research focuses on system design methodologies, traffic management system software, and advanced traffic signal controller (ATC). $\mathrm{He}$ is the Chair of the National Academies - Transportation Research Board's Traffic Signal Systems Committee (AHB25), and a member of INCOSE, INFORMS, IEEE, IIE and ITE. He can be reached at <larryesie.arizona.edu>. 